\title{
De Cajas de Ahorro a sociedades capitalistas y Fundaciones: un nuevo enfoque en la gestión y aplicación de la Obra Social
}

\section{Carlos Aranda Ruiviejo y Eva Sotomayor Morales}

RESUMEN: El presente trabajo se ha desarrollado con la finalidad de analizar las estrategias emprendidas por las nuevas Fundaciones surgidas tras el proceso de reestructuración del sector de las Cajas de Ahorro para garantizar la sostenibilidad de la Obra Social que anteriormente venían realizando estas entidades. Para ello se realiza un estudio de carácter descriptivo y explorativo que comprende un horizonte temporal de diez años y en el que se contrastan planteamientos anteriores y posteriores a los procesos de integración, centrando el interés en la evolución del modelo de gestión y en las fuentes de financiación utilizadas para dotar de fondos a la partida presupuestaria dedicada a fines sociales. Una vez realizadas las valoraciones se evidencia una estrategia de Obra Social mucho más diferenciada, gestionada en su totalidad mediante la modalidad de Obra Propia y orientada hacia unos colectivos más específicos mediante actividades integradas en su mayor parte dentro del área de Asistencia Social. Todos estos cambios muestran una Obra Social financieramente más pequeña, pero más autosuficiente en su modelo de negocio y menos dependiente de los recursos de las entidades de crédito en las que participan como accionistas, incrementándose los ingresos por actividades propias y los derivados de alianzas mantenidas con instituciones público/privadas.

PALABRAS CLAVE: Fundaciones, Cajas de Ahorro, Obra Social, Sostenibilidad.

CLAVES ECONLIT: D53, M14, M21.

Cómo citar este artículo / How to cite this article: ARANDA, C. \& SOTOMAYOR, E. (2017): "De Cajas de Ahorro a sociedades capitalistas y Fundaciones: un nuevo enfoque en la gestión y aplicación de la Obra Social", CIRIEC-España, Revista de Economía Pública, Social y Cooperativa, 91, 149-173.

Correspondencia: Carlos Aranda Ruiviejo, Universidad de Jaén, e-mail: caruiviejo@gmail.com, y Eva Sotomayor Morales, Universidad de Jaén, esotoma@ujaen.es. 


\section{EXPANDED ABSTRACT}

\section{From Saving Banks to Capitalist Societies and Foundations: a new approach on the managing and implementation of the Social Work}

This work has been carried out with the aim of analyzing the strategies undertaken by the new Foundations emerged as a result of the process of restructuration in the sector of the Savings Banks (SB), having such process the purpose of ensuring the sustainability of the Social Work (SW) that was traditionally made by those entities. Since the beginning of the international financial crisis in August 2007 , a process of global economic recession starts with direct and adverse consequences to the real economy of those countries that had registered constant economic growth rates until then.

In Spain all these macroeconomic changes have affected mainly to the SBs, starting at the beginning of 2010 a process of reordering leading to a complete rearrangement of their legal nature and the aim they were intended. As a result, out of the 45 SBs existing at the beginning of 2010, only two of them continued operating in December 2016 as such (CaixaOntinyent y CaixaPollença), being the rest integrated in two private banks (BBVA y Banco Sabadell) and eight entities with a bank statute in charge of the financial performance (Kutxa, Liberbank, BMN, Ibercaja, Abanca, CaixaBank, Unicaja y Bankia).

Similar to what had been observed in other countries such as Italy or the United Kingdom, the Law 26/2013, 27 December, ruling the Savings Banks and Bank Foundations (Ley 26/2013, de 27 de diciembre, de Cajas de Ahorros y FundacionesBancarias (LCAFB))sets a historical landmark to this type of credit entities, separating the financial from the social activity and introducing a new type among the foundational institutions: Bank Foundations. It is pretended with this not only to manage the participation as stakeholders in the participated financial entity, but also the SW that traditionally was developed by the SBs.

In this context, the financial business is administrated by an anonymous society with a bank statute, and the benefic-social activity is handled by Bank Foundations (BFs) or Ordinary Foundations (OFs) defined by the amount of participation in the financial entity, and being in charge of managing the politics of social interest. Consequently, in 2016 the SW was materialized by fourteen BFs, nineteen OFs and one special Foundation, emerging as a result of the sectoral restructuring process. 


\section{DE CAJAS DE AHORRO A SOCIEDADES CAPITALISTAS Y FUNDACIONES: UN NUEVO ENFOQUE EN LA GESTIÓN Y APLICACIÓN DE LA OBRA SOCIAL}

Following this scenario, this investigation sets a descriptive and explorative study to analyze the structure and funding of the SW during the period of 5 years previous to the sectoral restructuring process (2006-2010) and its contrast with the new social approach resulting from the transformation of the SBs into BFs or OFs (2011-2015), and consequently aiming to establish a general overview of the new configuration of the sector and to identify the current management strategies from the economic and social point of view.

In so doing, two differentiated analyses are conducted. On one hand, a financial study of the SW is carried out to evaluate the evolution of the budgetary resources destined to finance social activities and its funding sources, analyzing in detail the new monetary flows arisen as a result of the process of sectoral restructuration and its implication in the business strategy. On the other hand, the management models applied to the SW and the progression of the different social investment areas are examined.

The legislative and sectoral analysis reveals some issues that substantially affect the SBs model traditionally known until the beginning of restructuration process in 2010. It is evidenced a deep fragmentation of the sector that affect the most characteristic elements of this type of financial institutions: the SW and its Corporate Government.

Under the traditional model, the SBs traditionally had a similar pattern in everything related to its government structure, surpluses distribution, management of the SW and its social policy. Apart from exceptions, in the decision-making bodies would participate the same groups of interest and practically the same amounts of money were destined to finance the SW, being the Saving Bank in charge of financing it almost completelyby means of the modality of Own Work or Collaborative Work, preferentially through two lines of social investment: Culture and Free Time, and Social Assistance.

Once the LCAFB came into force, two types of institutions emerged to apply the social policy (BFs or OFs), existing significant differences between their business model. Firstly, considering their decision-making bodies, it can be observed a difference between their legislative frameworks, the Foundations Law for the OFs and the LCAFB for the BF, leading to the creation of heterogeneous government structures of the Foundations. A greater surveillance on the configuration of the decision-making bodies has been established in the BFs, existing a lack of control on the OFs, and thus an absence of regulation over the groups of interest that can be a member, the transparency of the entities that freely publish annual reports mentioning their corporate government, and the investment policy.

Similarly, from the social point of view,important inequalities can be found depending on the Foundation, a priori having more advantages the biggest entities with a significant participation in the credit entity. However, only 6 out of the 34 Foundations linked to Banks present a shareholding higher than the $25 \%$ of the capital, receiving the rest of them an insignificant or null contribution in the financial entity. 
Thus, only a few Foundations perceive important monetary flows from the financial entities, and on the contrary, a notorious number of Foundations have an insignificant or null dividend distribution. Therefore, each entity will establish differentiated strategies based on their financial needs, being predictable the biggest BFs to carry out the most relevant social projects.

With this context, the smaller OFs must take benefit from other competitive advantages such as the specialization on specific regions, agreements with public/private institutions, and their own activities developed through social, cultural and sport centers, cultural heritage and pawnshops. So far, some Foundations have positioned in such way, resulting a SW financially much smaller compared to the period previous to the sectoral restructuration process, fully managed through the modality of the Own Work, and oriented to much more specific collectives through the Social Assistance area. The high assistance costs can be the result of optimizing the social demands with a service offer that needs to be efficient in a scenario where the monetary flows dedicated to this type of activities have been considerably reduced.

KEYWORDS: Foundations, Saving Banks, Social Work, Sustainability. 


\section{1.- Introducción}

Con el comienzo de la crisis financiera internacional en agosto de 2007 se da inicio a un proceso de recesión económica de carácter global con unas consecuencias directas y adversas en la economía real de aquellos países que habían experimentado tasas de crecimiento constantes hasta ese momento. El inicio de la crisis en el mercado hipotecario estadounidense como consecuencia de la insolvencia generada por los créditos de alto riesgo (subprime), da lugar a que se establezca un escenario de desconfianza e inestabilidad en los mercados financieros de todo el mundo.

Tales circunstancias se agravan con la quiebra de Lehman Brothers en septiembre de 2008, el carácter global de la entidad financiera y la estrecha interconexión con los principales Bancos mundiales ocasionaría una profunda conmoción en los mercados de valores de las principales economías. La inestabilidad financiera empieza a mostrar evidencias de una serie de desequilibrios que afectan especialmente a la gestión de riesgos, los mecanismos de supervisión y regulación, y las agencias de rating que se han mostrado incapaces de valorar productos de un complejo entramado financiero, encontrándose diferentes niveles de impacto en función del país que tomemos en consideración.

Las diferentes regulaciones normativas tanto de ámbito nacional como europeo han dado lugar a que se establezca un entorno institucional mucho más regulado tanto en términos de solvencia como de operatividad bancaria. Actualmente, a las nuevas exigencias de capital en los mercados financieros (Basilea III), hay que añadir el Mecanismo Único de Supervisión adoptado en noviembre de 2014 donde el Banco Central Europeo (BCE) asume la competencia para la supervisión bancaria de la zona euro.

En España, todos estos cambios macroeconómicos han afectado especialmente a un tipo de entidad financiera: las Cajas de Ahorro (CA), dándose inicio en 2010 a un proceso de reordenación bancaria en el que se modifica por completo la naturaleza jurídica y la finalidad con que éstas surgieron. Tales han sido las circunstancias que de 45 CA a comienzos de 2010, en diciembre de 2016 tan sólo dos de ellas continuaban operando como tal, pasando a integrarse el resto en un total de ocho Grupos Bancarios constituidos como Sociedades Anónimas (SA) y donde se da entrada a nuevos grupos de interés dentro de los órganos de decisión: accionistas ${ }^{1}$. 
De forma similar a lo ocurrido en otros países como Italia o Reino Unido, la Ley 26/2013 de 27 de diciembre de Cajas de Ahorros y Fundaciones Bancarias (LCAFB) ${ }^{2}$ supuso un nuevo hito en la historia de este tipo de entidades de crédito, separando la actividad financiera de la social e introduciendo una nueva figura entre las instituciones de tipo fundacional: las Fundaciones Bancarias. Con las mismas se pretende no sólo gestionar la participación como accionistas en la entidad financiera participada, sino también la Obra Social (OS) que anteriormente venían desarrollando las CA.

Todas estas disposiciones normativas han propiciado un nuevo enfoque en la gestión no sólo de la actividad financiera sino también de la social, surgiendo una serie de Fundaciones Bancarias u Ordinarias desde las que se diseña y ejecuta la OS. A partir de ahora, serán éstas las encargadas de planificar la estrategia social y de definir cuáles son las principales líneas de actividad, teniendo en cuenta que las posibilidades de financiación respecto a la entidad financiera se limitan al reparto de dividendos y a los acuerdos de colaboración que se establezcan con la misma.

Por todo ello, se presenta un escenario incierto en el devenir de unas entidades centenarias en programas de cohesión social y transformadas en sociedades capitalistas muy alejadas del fin social con el que surgieron en un principio, planteándose una serie de incógnitas que afectan a la actividad de su principal seña de identidad: la OS. Ante este contexto, en el presente trabajo de investigación se planteó un estudio descriptivo y exploratorio en el que se analiza la estructura y financiación de la OS durante los cinco años anteriores al proceso de reestructuración sectorial (2006-2010) y su posterior contraste con los nuevos planteamientos sociales tras la conversión de las CA en Fundaciones Bancarias u Ordinarias (periodo 2011-2015), todo ello con objeto de establecer una visión general sobre la nueva configuración del sector y de identificar las actuales estrategias de gestión en el plano económico y social.

Concretamente, se realizan dos análisis diferenciados: por un lado, se realiza un estudio financiero de la OS en el que se evalúa la evolución de los recursos presupuestarios destinados a financiar actividades sociales y sus fuentes de financiación, profundizando en los nuevos flujos monetarios surgidos tras el proceso de reestructuración sectorial y su implicación en la estrategia empresarial. Por otra parte, se efectúa una revisión sobre los modelos de gestión aplicados a la OS y sobre la progresión de las diferentes áreas de inversión social.

En su contenido, el resto del artículo se estructura como sigue: en primer lugar, se analiza la actividad social de las CA desde el origen de los Montes de Piedad hasta su conversión en Fundaciones Bancarias u Ordinarias. En segundo lugar, se presentan los objetivos y metodología del estudio. Posteriormente, se evalúan los datos obtenidos del análisis de cada una de las variables consideradas examinando un total de diez años. Finalmente, se exponen las conclusiones y referencias bibliográficas. 


\section{2.- La Obra Social en las Cajas de Ahorro: pasado y presente de una actividad pionera en políticas de desarrollo regional}

\subsection{La Obra Social bajo el modelo tradicional: una revisión de los principales pre- ceptos normativos}

Con carácter general, la mayor parte de la doctrina considera que el origen de las CA se encuentra vinculado a instituciones de tipo benéfico, especialmente con los Montes de Piedad. En España, el primer Monte de Piedad se constituyó en Madrid en 1702 por iniciativa del sacerdote Francisco Piquer, al inicio estas instituciones financiaban su actividad mediante la concesión de préstamos prendarios a bajo interés a las clases sociales más desfavorecidas y con las aportaciones realizadas por los fieles a cambio de oraciones en actos religiosos. En un principio la financiación de los Montes resultó ser muy variada (legados, rentas, limosnas, etc.), siendo con la llegada a España de las CA cuando se obtuvo un medio de financiación mucho más sólido y estable.

Realizando una revisión de los preceptos normativos, el antecedente más cercano sobre la regulación de las CA lo encontramos en la publicación de la primera norma conocida que afecta a este tipo de entidades: la Real Orden de 3 de abril de 1835, en ésta se hace referencia a su actividad reflejando que el menestral, el jornalero y todo hombre laborioso puede depositar sumas tenues de dinero bajo la confianza de obtener un crédito proporcionado, añadiendo que estas instituciones contribuyen en gran medida a propagar el espíritu de economía. Unos años más tarde, en el Decreto de Fundación y aprobación del Reglamento de la primera CA fundada en España de fecha 25 de octubre de 1838, la Reina gobernadora en nombre de su hija Isabel II declara lo conveniente que sería establecer en Madrid una CA en la que las clases menos acomodadas puedan depositar pequeñas cantidades a cambio de réditos con la facultad de retirarlos en cualquier momento.

Con todo ello, en 1838 se constituye en España la primera CA: Caja de Ahorros de Madrid, organizándose corporativamente como la unión entre el Monte de Piedad que operaba en la ciudad madrileña y la CA recién fundada. Unos meses más tarde se promulga la Real Orden de 17 de abril de 1839 donde se exhorta a los gobernadores civiles de toda España a que realicen los esfuerzos necesarios con el fin de establecer en cada provincia al menos una CA asociada a un Monte de Piedad.

Con la fundación de la CA de Madrid, el modelo Monte-Caja pronto se extendió a otros territorios peninsulares como Andalucía, Valencia, Cataluña, Cantabria o País Vasco, definiéndose su carácter benéfico con la entrada en vigor de la Ley de 29 de junio de 1880 que además añadió el protectorado oficial sobre éstas. Asimismo, se establecía la necesidad de que se mantuvieran unidos Monte y Caja para poder auxiliarse recíprocamente, destacando que las CA tendrán como fin principal auxiliar a las clases más necesitadas mediante la concesión de préstamos a un módico interés. 
Todas estas disposiciones normativas evidencian que desde su génesis en el siglo XIX el esfuerzo del legislador se ha centrado en mantener el tándem Monte-Caja, estructura que aún perdura en nuestros días. De este modo, en 2015 existían en España doce Montes de Piedad con veintidós oficinas repartidas por todo el territorio nacional, todos ellos vinculados a los Bancos y Fundaciones resultantes del proceso de reestructuración sectorial ${ }^{3}$.

El interés y la iniciativa pública por la creación de este tipo de entidades también tuvo su reflejo en el fuerte intervencionismo de su actividad económica y social, no siendo hasta la década de los setenta cuando las CA adquieren unas estructuras organizativas y funcionales que les permiten operar dentro de nuestro sistema financiero. Así, con la publicación de la Ley de Ordenación Bancaria de 1921 (Ley Cambó) y, el posterior Real Decreto de 9 de abril de 1926 se abre un nuevo periodo caracterizado por un fuerte intervencionismo público y de aislamiento en el sector financiero, limitando su operatoria frente a la Banca privada. Igualmente, se establece por primera vez un coeficiente de inversión obligatorio de al menos el 40\% del saldo de sus ahorros en Fondos Públicos del Estado.

Durante el gobierno franquista fueron varias las disposiciones promulgadas en las que se establecía el protectorado público por parte del Ministerio de Hacienda o el de Trabajo en función de los intereses del Estado de intervenir en la obra benéfico-social o en la actividad financiera de éstas. Así por ejemplo, en el Decreto de 17 de octubre de 1947 se fijan tres líneas de actuación social (instituciones sanitarias, culturales y construcción de viviendas protegidas) que deben ser financiadas por el $85 \%$ de los excedentes no destinados a reservas. Además, se establece que el $15 \%$ restante quedará a disposición del Ministerio de Trabajo para financiar obras benéfico-sociales de interés nacional.

En la misma línea se encuentra el Decreto de 9 de marzo de 1951 sobre inversiones obligatorias, en éste se establece la obligación de destinar como mínimo el $60 \%$ de los recursos ajenos totales a Fondos Públicos españoles admitidos a cotización oficial. Hechos éstos que ponen de manifiesto la estrecha relación entre la actividad de las CA y las necesidades de financiación del sector público.

Bajo este contexto, no sería hasta la segunda mitad del siglo XX cuando se desplace el carácter benéfico de su actividad y se establezca una nueva etapa en la que se equiparen funcionalmente al resto de entidades de crédito que operan en nuestro país. Con la publicación de la Ley de 14 de abril de 1962 sobre Ordenación del Crédito y la Banca se incluye a las CA en el Sistema Financiero, siendo con la llamada Reforma Fuentes Quintana (Orden Ministerial de 23 de julio de 1977, Reales Decretos 2290 y 2291/1977, de 27 de agosto, y Real Decreto 3047/1977, de 11 de noviembre) cuando se les otorga libertad operatoria en igualdad de oportunidades que la Banca privada, con libertad de expansión por todo el territorio nacional y la modernización de sus órganos de gobierno (Asamblea General, Consejo de Administración y Comisión de Control). 


\section{DE CAJAS DE AHORRO A SOCIEDADES CAPITALISTAS Y FUNDACIONES: UN NUEVO ENFOQUE EN LA GESTIÓN Y APLICACIÓN DE LA OBRA SOCIAL}

Con la entrada en vigor de la reforma Fuentes Quintana y con su nueva redacción en la Ley 31/1985 de 2 de agosto, de Regulación de las Normas Básicas sobre Órganos Rectores de las CA (LORCA), se configura definitivamente el modelo de CA que ha perdurado hasta su conversión en Bancos, encontrando dos elementos diferenciales que permiten distinguir a las CA del resto de instituciones que operan en nuestro sistema financiero: Gobierno Corporativo y OS.

Para el primer caso, se estableció que las CA al ser entidades de naturaleza fundacional y carácter social, en sus órganos de gobierno deberían estar representados los intereses de una pluralidad de grupos: (i) Impositores. (ii) Empleados. (iii) Entidades de Interés General. (iv) Entidades Fundadoras. (v) Corporaciones Locales y, (vi) Comunidades Autónomas (CCAA). La división del territorio nacional en un Estado de autonomías y las sentencias 48 y 49 de 1988 dictadas por el Tribunal Constitucional contribuyeron a otorgar un mayor poder de decisión a las CCAA en materia de CA, sobre todo por el carácter territorial que presentaba este tipo de entidades financieras. Todos estos factores dieron lugar a que se incrementara notablemente el peso de los poderes autonómicos tanto en los órganos de decisión como en las competencias asumidas para regular su actividad económica y social, desempeñando el doble papel de supervisor y supervisado.

Tales son las circunstancias, que si tomamos en consideración los datos fundacionales de las 45 CA existentes hasta el proceso de reestructuración sectorial, salvo tres Cajas que son propiedad de la Iglesia 4 , el resto presenta una titularidad predominantemente de carácter público. Por tanto, era el grupo perteneciente a los poderes públicos (Entidades Fundadoras, Corporaciones Locales y CCAA) el que ostentaba una mayor representación dentro de los órganos de decisión, con tasas de participación que en la mayor parte de los casos se situaban en torno al cincuenta por ciento del total de los intereses representados.

En nuestro Derecho interno sobre el carácter público o privado de las CA ya se pronunció el Tribunal Constitucional en su Sentencia 18/1984 de 7 de febrero, que creo jurisprudencia sobre sentencias posteriores relacionadas con esta materia y en la que se puso de manifiesto que las CA fundadas por Corporaciones Locales no tienen la naturaleza de entes públicos sino entes de carácter social, encontrando investigaciones en las que se puede observar una relación directa entre el peso del sector público y el riesgo de las entidades (Fonseca, 2005; Fonseca y González, 2005; Illueca et al. 2011), otras en las que se establecen relaciones negativas de eficiencia (Azofra y Santamaría, 2004), e incluso estudios en los que se trata de determinar su relación con el crédito concedido a las Administraciones Públicas (Melle y Maroto, 1999; García y Surroca, 2008).

La fuerte participación pública que presentaba la mayor parte del sector en el caso de que su fundador perteneciera a un ente público ha llevado a que en muchos casos las CA sean consideradas como "empresas públicas", y así lo defienden entre otros Boix y Ureña (2006) que justifican su integración

4.- CajaSur fue fundada por el Excelentísimo Cabildo Catedral de Córdoba como Monte de Piedad el día 1 de septiembre de 1864, la CAI fue fundada en 1905 por Acción Social Católica de Zaragoza y Cajacírculo fue constituida por el consejo de gobierno del Círculo Católico de Obreros de Burgos a instancia del Arzobispo de Burgos en agosto de 1908. 
dentro del sector público autonómico por cuatro razones: (i) el control público de los órganos de gobierno; (ii) el control público de las decisiones sobre el tamaño de las CA; (iii) las intervenciones administrativas en la actividad empresarial; y (iv) el control por la gestión y administración de la OS.

Dadas las circunstancias, tanto el Real Decreto-ley 11/2010, de 9 de julio, de órganos de gobierno y otros aspectos del régimen jurídico de las Cajas de Ahorros, como la LCAFB se centraron en establecer preceptos normativos que entre otras cosas limitaran la participación pública en los órganos de decisión de las $\mathrm{CA}$ en el caso de que la entidad fundadora perteneciera a un ente público, restringiendo su participación al $40 \%$ del total de los intereses representados en el primer caso y, al $25 \%$ en el caso de la LCAFB. Además, como señala Ureña (2015) la conversión de algunas de las CA más importantes en SA de ámbito y titularidad estatal las ha alejado casi definitivamente del poder político autonómico.

Por otra parte y en línea con lo anteriormente expuesto, el segundo elemento diferencial de las CA respecto a otro tipo de instituciones financieras lo encontramos en la dotación de una partida presupuestaria destinada a financiar la OS. Bajo el modelo tradicional, las CA no se conciben como entidades capitalistas cuya propiedad se encuentra diluida entre un elevado número de accionistas, sino como entes de naturaleza fundacional, por ello el reparto de excedentes se realiza de la siguiente forma: (i) Pago de Impuestos. (ii) Constitución de reservas para reforzar su capitalización y solvencia. (iii) Obra Social.

Dentro del estudio de la OS debemos de referirnos a dos aspectos básicos; su dotación presupuestaria y las diferentes líneas de inversión social que integran la misma. Para el primer caso, la Ley 44/2002 de Ordenación Económica y Medidas de Reforma del Sistema Financiero establece que una vez destinados a reservas un 50\% como mínimo de los excedentes de libre disposición, la parte restante puede dedicarse a un fondo orientado a financiar la OS. Por tanto, las CA tenían potestad para dedicar hasta el $50 \%$ de los excedentes de libre disposición a financiar sus actividades sociales y culturales.

Si tomamos como referencia las memorias de Responsabilidad Social Corporativa (RSC) de la Confederación Española de Cajas de Ahorro (CECA) en los años anteriores al proceso de reestructuración sectorial, podemos observar, que con carácter general en el sector se ha destinado de media un $75 \%$ de los excedentes de libre disposición a constituir reservar y un $25 \%$ a financiar la OS, evidenciando con ello una prioridad sobre la capitalización de las Cajas en detrimento de la actividad social. Este comportamiento parece comprensible si tenemos en cuenta que hasta su integración en Bancos las únicas fuentes de financiación provenían de la constitución de reservas y de la emisión en algunos casos de Deuda Subordinada, existiendo estudios empíricos que muestran una relación negativa entre la inversión social y las variables de performance 5 . 


\section{DE CAJAS DE AHORRO A SOCIEDADES CAPITALISTAS Y FUNDACIONES: UN NUEVO ENFOQUE EN LA GESTIÓN Y APLICACIÓN DE LA OBRA SOCIAL}

Las peculiaridades que presentan las CA en sus estructuras de gobierno, distribución de excedentes y por ende, en su modelo de negocio, parece ser que las ha situado en una posición de desventaja respecto a sus competidores más directos; la Banca privada. Trabajos como el realizado por Maudos (2001) ponen de manifiesto tal circunstancia, evidenciando unos mejores resultados en el caso de los Bancos debido en parte a la mayor cuota de mercado que éstos presentan.

En el plano de la eficiencia, las últimas investigaciones realizadas dentro del sector parecen confirmar esta hipótesis, mostrando mayores cuotas de rentabilidad para el caso de la Banca privada (Torres et al. 2012; Escobar y Guzmán, 2010; Guzmán y Reverte, 2008; Climent, 2012; Trujillo-Ponce, 2013; Climent y Pavía, 2014). Tal vez, éstas sean las razones que justifican la nueva realidad del sector donde se separa la actividad financiera de la social y en el que se apuesta por un modelo Stockholders como vía de desarrollo y competitividad.

Por su parte, la OS se ha venido aplicando mediante la modalidad de Obra Propia (OP) u Obra en Colaboración (OC) a través de cuatro líneas de inversión social: (i) Cultura y Tiempo Libre. (ii) Educación e Investigación. (iii) Asistencia Social y Sanidad. (iv) Patrimonio Histórico y Medio Ambiente. Esta forma de reparto quedó definida en el artículo 22 del Decreto 2290/1977, en el que se establece que: "las Cajas de Ahorros destinarán la totalidad de los excedentes que conforme a las normas vigentes no hayan de integrar sus reservas a la financiación de obras benéfico-sociales propias o en colaboración, de modo que las mismas se orienten hacia la sanidad pública, la investigación, enseñanza y cultura o los servicios de asistencia social y que los beneficios de ella derivados se extiendan especialmente en el ámbito regional de actuación de la Caja".

Durante años el impacto económico y asistencial de este tipo de iniciativas ha sido incuestionable, estudios como el realizado por Balado (2006) muestran que en 2004 el gasto promedio por habitante en OS fue de 26,94 euros, siendo Cultura y Asistencia Social las dos grandes áreas de referencia, alcanzando una cobertura del $53 \%$ de los municipios y del $96 \%$ de la población española, siendo ésta total en los municipios de más de 100.000 habitantes y superior al $80 \%$ de la población en aquellos municipios de más de 1.000 habitantes. Por tanto, su complementariedad con la labor pública en materia social parece evidente, estableciéndose diferentes prioridades estratégicas en función de las necesidades a cubrir en cada caso6:

- Década de los 70: el área Docente y Asistencial recibe la mayor parte del presupuesto para OS.

- Desde los 80 hasta 2001: el área Cultural se posiciona como la principal línea de inversión social con unas cantidades en torno al $45-50 \%$ del total presupuestado.

- 2002-2011: el área Cultural va perdiendo peso progresivamente en detrimento de Asistencia Social que pasa a situarse en 2009 en la principal línea de gasto. 


\subsection{Las Fundaciones Bancarias y Ordinarias: un nuevo referente entre las insti-} tuciones de economía social

De forma similar a lo ocurrido en otros países como Italia o Reino Unido, con la entrada en vigor de la LCAFB se lleva a cabo la mayor reforma del régimen jurídico y funcional de las $C A$ prácticamente desde su constitución, segregando la actividad financiera de la social e introduciendo diferentes figuras jurídicas desde las que se aplica su modelo de negocio. Bajo este contexto, el negocio bancario pasa a ser administrado por una SA con estatuto de Banco y la actividad benéfico-social queda en manos de unas Fundaciones que vienen definidas por el grado de participación en la entidad financiera y que son las encargadas de gestionar las políticas de interés social ${ }^{7}$.

En su desarrollo, la Ley se estructurada en dos títulos; en el primero de ellos se aborda la regulación propia de las CA mientras que en el segundo se recogen las medidas referentes a las Fundaciones resultantes de los procesos de integración. Así, para las CA que decidan seguir operando como tal la normativa introduce una serie de reformas que afectan tanto al ejercicio de su actividad como a los miembros de sus órganos de gobierno. De este modo, se exige que las CA desarrollen su actividad en el ámbito local y tengan un tamaño reducido, estableciendo que aquellas que crezcan por encima de los límites permitidos deberán transformarse en Fundaciones Bancarias, perdiendo su licencia bancaria y transmitiendo su actividad financiera a una entidad de crédito.

Por otro lado se apuesta por una mayor profesionalización de los órganos de gobierno, exigiendo que todos los integrantes del Consejo de Administración cuenten con conocimientos y experiencia para el ejercicio de sus funciones. Asimismo, se reduce el porcentaje de participación pública al 25\% del total de los intereses representados, dando un mayor protagonismo al grupo formado por los Impositores que pasan a tener una representación que oscila entre un mínimo del 50 y un máximo del 60 por ciento. Además, se incluye un nuevo grupo en los órganos de gobierno y comités de las Cajas: los consejeros independientes, todo ello con objeto de aportar neutralidad y objetividad en la toma de decisiones.

En segundo lugar, se establece que aquellas $C A$ que reúnan los requisitos del art. 34.2 de la LCAFB (Activo total consolidado según el último balance auditado supere los 10 millones de euros 0 que su cuota en el mercado de depósitos de su ámbito territorial de actuación sea superior al $35 \%$ del total de depósitos), deberán transformarse en Fundaciones Bancarias u Ordinarias a cambio de acciones en el capital de un Banco al que traspasarán todo el patrimonio afecto a su actividad financiera. Para ello, la Ley diferencia entre Fundaciones Bancarias (FB): "aquellas que mantengan una participación en una entidad de crédito que alcance, de forma directa o indirecta, al menos, un 10 por ciento del capital o de los derechos de voto de la entidad o que le permita nombrar o destituir algún miem-

\footnotetext{
7.- Para analizar en profundidad la nueva Ley de Fundaciones Bancarias y los retos a los que se enfrentan las nuevas entidades, véase Emparanza (2015).
} 


\section{DE CAJAS DE AHORRO A SOCIEDADES CAPITALISTAS Y FUNDACIONES: UN NUEVO ENFOQUE EN LA GESTIÓN Y APLICACIÓN DE LA OBRA SOCIAL}

bro de su órgano de administración", y Fundaciones Ordinarias (FO), en caso de que la participación en la entidad de crédito no alcance el porcentaje citado anteriormente o que no puedan nombrar o destituir algún miembro de su órgano de administración.

Con este marco reglamentario y tras la adaptación de las distintas entidades a los requisitos de la LCAB, en diciembre de 2016 nos encontramos ante un escenario en el que tan sólo coexisten dos CA de reducida dimensión (Caixa Ontinyent y Caixa Pollença), integrándose el resto entre dos Bancos privados (BBVA y Banco Sabadell) y ocho entidades con estatuto de Banco que son los encargados del desempeño financiero (Kutxa, Liberbank, BMN, Ibercaja, Abanca, CaixaBank, Unicaja y Bankia). Por su parte, la OS se materializa a través de catorce FB, diecinueve FO y una Fundación Especial (FE), entidades éstas surgidas tras el proceso de reestructuración.

Por tanto, a partir de ahora la tradicional OS de las CA se aplicará principalmente a través de dos tipos de Fundaciones (Bancarias u Ordinarias) que tendrán diferente grado de participación en el Banco desde el que se desarrolla la actividad financiera, hecho este que originará diferentes flujos de capital en función del valor accionarial en la entidad principal. Asimismo, debemos de considerar el régimen jurídico a aplicar en cada caso, dándose circunstancias que pueden dar lugar en un futuro mediato a estructuras de gobierno heterogéneas en función del tipo de Fundación que tomemos en consideración.

De este modo, en la LCAFB se establece que las FB quedarán sujetas a su régimen jurídico y con carácter supletorio a la Ley 50/2002 de 26 de diciembre de Fundaciones, o la normativa autonómica que resulte de aplicación. En cambio, no hace referencia expresa a las FO, quedando sometidas únicamente a la Ley de Fundaciones citada anteriormente. Por ello, se evidencia una mayor laxitud que puede afectar no sólo a la transparencia de las FO, sino también a las estructuras de su gobierno corporativo.

Como se desprende de la revisión normativa, en la LCAFB se establecen una serie de obligaciones que conciernen únicamente a las FB, entre éstas, se implantan una serie de obligaciones financieras (planes financieros y fondos de reserva) para aquellas Fundaciones cuya participación en la entidad de crédito exceda del 30 y 50 por ciento respectivamente. También, se expresa la necesidad de hacer público con carácter anual un informe de gobierno corporativo en el que se reflejen aspectos como la estructura de gobierno o la política de inversión de las entidades. Además, se regula tanto la composición como los requisitos que deben de reunir los miembros que formen parte del principal órgano de gobierno de las Fundaciones: el Patronato.

Por tanto, si contrastamos lo que regula la LCAFB en materia de gobierno corporativo con lo que establece la Ley 50/2002 de Fundaciones, podemos observar que para las FB se establecen una serie de requisitos e incompatibilidades para ser miembro del Patronato, se limita la participación pública al $25 \%$ del total de los intereses representados y, además, se fijan los siguientes grupos de participación: (i) Personas o entidades fundadoras. (ii) Entidades representativas de intereses colectivos del ámbito 
de actuación de la Fundación. (iii) Personas privadas, físicas o jurídicas que hayan aportado de manera significativa recursos a la Fundación. (iv) Personal independiente de reconocido prestigio profesional en la actividad social. (v) Personal que posea conocimientos y experiencia específica en materia financiera.

En cambio, en el artículo 15.2 de la Ley de Fundaciones tan sólo se establece que podrán ser miembros del Patronato las personas físicas que tengan plena capacidad de obrar y no estén inhabilitadas para el ejercicio de cargos públicos, recogiendo además que las personas jurídicas podrán designar a las personas físicas que las representen. Por tanto, parece evidente que en un principio el mayor interés se ha centrado en aquellas FB que poseen una participación significativa en el Banco desde el que se desempeña la actividad financiera.

\section{3.- Metodología y objetivos}

Como se expuso en los párrafos precedentes, con este estudio se pretende identificar en qué medida ha afectado a la OS de las CA el proceso de reestructuración sectorial que se inició a mediados 2010 y que culminó a finales de 2014 con la desaparición de estas entidades y con la creación de nuevas sociedades donde se separa la actividad financiera de la social, dándose entrada a las Fundaciones Bancarias u Ordinarias como instituciones encargadas de gestionar la política social.

Tales circunstancias plantean una serie de cuestiones que creemos necesario analizar. De este modo, sería interesante examinar en qué medida está evolucionando la OS de las extintas CA bajo la dirección de las Fundaciones, instituciones en las que se reduce considerablemente el peso del sector público dentro de los órganos de decisión y donde se da entrada a personal externo con conocimientos y experiencia en el ámbito social y financiero, lo que hace previsible una gestión más profesionalizada del modelo de negocio.

Igualmente, sería interesante analizar la política financiera utilizada por las nuevas Fundaciones para dar continuidad a la antigua OS de las CA. Bajo este nuevo modelo no se cede una parte del beneficio neto a tal finalidad, sino que las principales fuentes de financiación provienen de las actividades propias y de los flujos monetarios derivados del reparto de dividendos, encontrando diferentes grados de participación en las entidades financieras dependiendo del accionariado de las FB o FO.

Todas estas cuestiones plantean una serie de retos que cambiarán sin duda la forma de proceder de estas instituciones centenarias en políticas de desarrollo regional. Ante esta realidad, se ha decidido realizar un estudio donde se analiza la situación previa de la OS hasta el proceso de restructu- 


\section{DE CAJAS DE AHORRO A SOCIEDADES CAPITALISTAS Y FUNDACIONES: UN NUEVO ENFOQUE EN LA GESTIÓN Y APLICACIÓN DE LA OBRA SOCIAL}

ración sectorial de 2010 y, contrastarlo posteriormente con los nuevos planteamientos tras su conversión en Fundaciones. Para ello, se ha tomado en consideración un horizonte temporal de diez años que se divide en dos fases diferenciadas: (i) 2006-2010. Periodo en el que la OS es gestionada según el modelo tradicional de las CA. (ii) 2011-2015. Horizonte temporal en el que se empiezan a materializar los procesos de integración y donde las Fundaciones son las encargadas de gestionar la política social.

Para alcanzar los objetivos planteados se ha seleccionado una muestra no probabilística donde se toma en consideración los datos obrantes en la CECA de las 45 CA que integraban el sistema financiero español hasta el proceso de reestructuración sectorial y, todas las entidades resultantes tras la entrada en vigor de la LCAFB.

Al compararse estrategias empresariales ante diferentes contextos normativos e institucionales, creemos que lo más conveniente sería plantear un análisis descriptivo y exploratorio basado en el análisis de contenido tanto de carácter cuantitativo como cualitativo, siendo los datos publicados por la CECA la principal fuente de recogida de evidencias, especialmente los referidos a los Anuarios Estadísticos, OS, Responsabilidad Social, Montes de Piedad y Gobierno Corporativo. En base a los planteamientos anteriormente expuestos, se buscó alcanzar los siguientes objetivos:

- Evaluar en qué medida han evolucionado las distintas áreas de inversión en que se divide la OS.

- Analizar los efectos de la crisis económica en el gasto social y su implicación en el modelo de gestión.

- Examinar la política financiera de la OS y su importancia en la estrategia empresarial

\section{4.- El papel de las Fundaciones en la continuidad de la Obra Social: ¿riesgo u oportunidad?}

Para dar respuesta a los planteamientos anteriormente expuestos, se realizó un análisis relacionado con la actividad de la OS en años anteriores al proceso de reestructuración del sector de las CA y su posterior contraste con las iniciativas llevadas a cabo una vez que se empiezan a materializar los procesos de integración y que finalizan con la conversión de las CA en Fundaciones.

En cada uno de los periodos evaluados se realizan dos estudios diferenciados; por un lado, se lleva a cabo un análisis financiero de la OS donde se revisan las tendencias y valores medios tanto de la cantidad de recursos que se dedican a financiar la OS como de sus fuentes de financiación, deta- 
llando aquellas líneas de ingreso que son relevantes para la sostenibilidad de las iniciativas a largo plazo. Por otro lado, se realiza una revisión sobre la evolución de las distintas áreas de inversión en que se divide la OS de las CA, especificando los modelos de gestión que se utilizan para aplicar la totalidad de recursos y su repercusión en la estrategia empresarial.

En línea con lo anteriormente expuesto, cabe mencionar que hasta el inicio del proceso de reestructuración sectorial la OS se aplicaba principalmente a través de cuatro áreas: (i) Cultura y Tiempo Libre; (ii) Asistencia Social y Sanidad; (iii) Educación e Investigación; y (iv) Patrimonio Histórico y Medio Ambiente. No obstante, en el ejercicio 2013 se lleva a cabo una reestructuración de áreas con el fin de adaptarlas a las demandas sociales, pasando éstas de cuatro a seis: (i) Acción Social; (ii) Cultura y Patrimonio; (iii) Educación e Investigación; (iv) Medio Ambiente; (v) Desarrollo Local y Creación de Empleo; y (vi) Deporte y Ocio. En nuestro estudio, con el fin de unificar criterios y de hacer comparables las distintas áreas en cada uno de los periodos evaluados se siguió la clasificación de cuatro áreas, incluyendo dentro del área de Patrimonio y Medio Ambiente para el periodo 2013-2015 las partidas correspondientes a Medio Ambiente, Desarrollo Local y Creación de Empleo y Deporte y Ocio, pertenecientes éstas a la nueva estructura de gasto que integra la actual OS.

\subsection{Gestión y distribución del gasto social para el periodo 2006-2015}

En la Tabla 1 se muestra la evolución de las distintas áreas de inversión social a lo largo del periodo evaluado, diferenciando entre la cantidad de recursos que son gestionados mediante la propia entidad (OP) y aquella en la que se aportan flujos monetarios para colaborar con otras instituciones púbico/privadas $(\mathrm{OC})$.

En el análisis de la distribución de gasto social para el periodo 2006-2010, se observa que de media se han destinado cantidades prácticamente similares tanto en el área de Cultura y Tiempo Libre como de Asistencia Social, evidenciándose un mayor repunte de esta última a partir del ejercicio 2008. El tercer puesto en inversión lo ocupa Educación e Investigación, siendo el área de Patrimonio y Medio Ambiente la que menos recursos recibe durante este ciclo de actividad.

Si hacemos extensivo el estudio al número de beneficiarios, es el área de Cultura y Tiempo Libre la que destaca por encima de todas las demás, siendo Asistencia Social la que ocupa el segundo lugar con unos valores notoriamente inferiores a la primera. Concretamente, en 2006 el número de beneficiarios de Cultura y Tiempo Libre fue de 64.496.938 frente a los 26.621.537 de Asistencia Social. Igualmente, en 2010 estas cantidades se situaban en 56.532 .905 para la primera y 29.304 .473 para la segunda 8 . 
Tabla 1. Modelo de gestión y distribución del gasto para el periodo 2006-2015 (\%)

\begin{tabular}{|c|c|c|c|c|c|c|}
\hline Cultura y & 2006 & 2007 & 2008 & 2009 & 2010 & Media \\
\hline Tiempo & 39,96 & 36,75 & 35,43 & 33,15 & 30,8 & 35,1 \\
\hline \multirow{2}{*}{ Libre } & 2011 & 2012 & 2013 & 2014 & 2015 & Media \\
\hline & 32,3 & 28,95 & 18,86 & 20,03 & 17,43 & 23,5 \\
\hline Asistencia & 2006 & 2007 & 2008 & 2009 & 2010 & Media \\
\hline Social y & 32,09 & 37,32 & 37,95 & 40,86 & 45,6 & 38,7 \\
\hline \multirow[t]{2}{*}{ Sanidad } & 2011 & 2012 & 2013 & 2014 & 2015 & Media \\
\hline & 43,5 & 48,39 & 47,16 & 50,29 & 45,43 & 46,9 \\
\hline Educación e & 2006 & 2007 & 2008 & 2009 & 2010 & Media \\
\hline \multirow[t]{3}{*}{ Investigación } & 17,22 & 16,30 & 15,87 & 17,30 & 16 & 16,5 \\
\hline & 2011 & 2012 & 2013 & 2014 & 2015 & Media \\
\hline & 16,9 & 17,15 & 14,43 & 11,11 & 11,73 & 14,3 \\
\hline Patrimonio & 2006 & 2007 & 2008 & 2009 & 2010 & Media \\
\hline Histórico y & 10,73 & 9,62 & 10,74 & 8,69 & 7,5 & 9,7 \\
\hline \multirow[t]{2}{*}{ Medio Ambiente } & 2011 & 2012 & 2013 & 2014 & 2015 & Media \\
\hline & 7,3 & 5,51 & 19,55 & 18,57 & 25,41 & 15,3 \\
\hline \multicolumn{7}{|c|}{ Modelo de gestión } \\
\hline Año & 2006 & 2007 & 2008 & 2009 & 2010 & Media \\
\hline Obra propia & 62,82 & 63,92 & 62,2 & 63,1 & 66 & 63,6 \\
\hline Obra en colaboración & 37,18 & 36,08 & 37,8 & 36,9 & 34 & 36,4 \\
\hline Año & 2011 & 2012 & 2013 & 2014 & 2015 & Media \\
\hline Obra propia & 67,8 & 69 & 100 & 100 & 100 & 87,4 \\
\hline Obra en colaboración & 32,2 & 31 & 0 & 0 & 0 & 12,6 \\
\hline
\end{tabular}

FUENTE: Elaboración propia. 
Durante este periodo, la fuerte inversión en actividades culturales y de tiempo libre parece evidenciar que se apuesta por una estrategia de OS de carácter general, orientada hacia un elevado número de beneficiarios y con la que se pretende reforzar la notoriedad e imagen corporativa de las Cajas. En la gestión se observa que aunque el modelo predominante ha sido el de OP, también se dedican importantes cantidades a financiar actividades mediante la OC, situándose la media para este periodo en el $36,4 \%$ de la partida de gasto.

Por su parte, durante el segundo periodo evaluado se observa que desde el ejercicio 2012 el área de Cultura y Tiempo Libre va reduciendo progresivamente su peso sobre el total de inversión hasta situarse en 2015 en el 17,43\% del gasto social, dato significativo si tenemos en cuenta que durante el periodo previo al proceso de reestructuración esta partida ocupaba una de las principales áreas de inversión. En cambio, Asistencia Social se consolida como la principal línea de gasto tomando valores en torno al $50 \%$ del total presupuestado. Asimismo, cabe destacar la creación de nuevas partidas sociales como Desarrollo Local y Creación de Empleo que en nuestro estudio se incluyen dentro de Patrimonio y Medio Ambiente y que en 2015 tuvieron una inversión total del 12,5\%.

Con la nueva configuración de gasto podemos apreciar que se apuesta por una OS mucho más específica. En este caso, no se invierte tanto en actividades culturales y de tiempo libre orientadas hacia un elevado número de beneficiarios, sino que se dirige hacia actividades sociales o de desarrollo que incluyen colectivos más específicos ${ }^{9}$. En concreto, durante el periodo 2013-2015 el número de beneficiarios pertenecientes a colectivos específicos representaban el $55,19 \%, 59,25 \%$ y $62,41 \%$ respectivamente ${ }^{10}$.

Bajo la dirección de las nuevas Fundaciones se observa que el modelo de gestión se orienta únicamente hacia la modalidad de OP, siendo éstas las encargadas de dirigir, gestionar y administrar los centros desde los que materializan las actividades sociales. Con ello, se desvinculan de la actividad financiadora y apuestan únicamente por acciones propias que les permitan ser eficientes dentro del mercado en el que operan.

\subsection{Análisis financiero de la OS bajo la modalidad de Cajas de Ahorro y Fundaciones}

Durante el periodo 2006-2010, las CA una vez que constituyen reservas y que realizan el pago de impuestos destinan la parte restante del beneficio neto a un fondo destinado a financiar la OS. A partir del ejercicio 2012 las Fundaciones se desvinculan más de la entidad de crédito y aparecen nuevas fuentes de financiación, siendo sus principales magnitudes las que se exponen a continuación:

9.- La mayor inversión se orienta hacia Infancia y Juventud, mayores de 65 años, personas en riesgo de exclusión, personas con necesidades especiales y emprendedores.

10.- Véase Memoria de Obra Social 2013, 2014 y 2015. CECA. Madrid

CIRIEC-España, Revista de Economía Pública, Social y Cooperativa

ISSN: 0213-8093

№91/2017, pp. 149-173 


\section{Tabla 2. Análisis financiero de la Obra Social para el periodo 2006-2015}

\begin{tabular}{|c|c|c|c|c|c|c|}
\hline Años & 2006 & 2007 & 2008 & 2009 & 2010 & Media \\
\hline $\begin{array}{l}\text { Dotación presupuestaria } \\
\text { (miles de } € \text { ) }\end{array}$ & 1.524 .629 & 1.824 .295 & 2.058 .971 & 1.775 .926 & 1.462 .366 & 1.729 .237 \\
\hline Años & 2011 & 2012 & 2013 & 2014 & 2015 & Media \\
\hline $\begin{array}{l}\text { Dotación presupuestaria } \\
\text { (miles de } € \text { ) }\end{array}$ & 1.124 .918 & 818.589 & 647.729 & 709.905 & 717.385 & 803.705 \\
\hline \multicolumn{7}{|c|}{ Fuentes de financiación (\%) } \\
\hline Años & 2006 & 2007 & 2008 & 2009 & 2010 & Media \\
\hline Aportación propia & 93,6 & 94,4 & 94,3 & 92,9 & 92,9 & 93,6 \\
\hline Ingresos por otras actividades ${ }^{11}$ & 6,4 & 5,6 & 5,7 & 7,1 & 7,1 & 6,4 \\
\hline Años & 2011 & 2012 & 2013 & 2014 & 2015 & Media \\
\hline Aportación propia & 87 & 75 & 18,42 & 20,87 & 21,57 & 44,6 \\
\hline Ingresos por otras actividades & 13 & 25 & 81,58 & 79,13 & 78,43 & 55,4 \\
\hline \multicolumn{7}{|c|}{ Desglose de los ingresos generados por otras actividades a partir de 2011 (\%) } \\
\hline Actividades propias & Ingresos & 12,10 & 12,85 & 33,01 & 36,05 & 23,5 \\
\hline Dividendos/Beneficios & de la & 77,76 & 66,32 & 35,44 & 32,70 & 53,08 \\
\hline Remanente de años & propia OS & & & & & \\
\hline anteriores y fondo para la OS & y subven- & 9,32 & 18,42 & 20,87 & 21,57 & 17,54 \\
\hline $\begin{array}{l}\text { Ayudas privadas, patrocinios } \\
\text { y contratos }\end{array}$ & $\begin{array}{c}\text { ciones } \\
\text { recibidas }\end{array}$ & 0,04 & 1,29 & 6,72 & 6,28 & 3,58 \\
\hline Subvenciones y ayudas púb. & & 0,78 & 1.12 & 3,96 & 3,40 & 2,3 \\
\hline
\end{tabular}

FUENTE: Elaboración propia.

En la Tabla 2 se pone de manifiesto el importante esfuerzo inversor que las CA han realizado en materia de OS, lo que da una idea del impacto económico y social en aquellas zonas donde se llevan a cabo las iniciativas. De este modo, durante la fase previa al proceso de reestructuración se destinó de media 1.729 millones de euros a políticas de desarrollo regional, ya sea en su vertiente social o cultural, siendo prácticamente la totalidad de estos recursos financiados íntegramente por las Cajas (93,6\%). Tan sólo un 6,4\% de los fondos provienen de actividades propias de la OS y de subvenciones recibidas en alianzas mantenidas con diferentes instituciones.

11.- Durante el periodo 2006-2010 todas las cantidades incluidas en este apartado se refieren principalmente a dos fuentes de financiación: las generadas por la propia OS en el desempeño de su actividad y las subvenciones recibidas de instituciones público/privadas. 
En cambio, durante el segundo periodo evaluado nos encontramos ante una OS financieramente mucho más pequeña que en el caso anterior, observando que los valores medios se reducen prácticamente a la mitad de la inversión (803 millones de euros), algo previsible si tenemos en cuenta el entorno de crisis y la disponibilidad de recursos respecto al Banco encargado de llevar a cabo la actividad financiera, limitándose ésta al reparto de dividendos como accionistas del mismo.

Como se desprende de la Tabla 2, el nuevo escenario normativo e institucional ha obligado a las Fundaciones a buscar fuentes de financiación alternativas para garantizar su sostenibilidad. Dentro de éstas, cabe destacar el notable crecimiento que ha registrado la partida de ingresos por actividades propias y en menor medida las de ayudas privadas y subvenciones, lo que muestra que la OS es cada vez más autosuficiente en su financiación y menos dependiente de la entidad de crédito. En este sentido, si durante el periodo previo al proceso de reestructuración la partida de ingresos por actividades propias obtuvo una media del 6,4\% del total financiado, en 2015 ésta representaba el 36,05\% del total, siendo el reparto de dividendos la segunda vía de ingresos de las Fundaciones $(32,7 \%)$.

Sin embargo, si profundizamos en la disposición de las principales fuentes de financiación una vez materializados los procesos de integración, observamos diferencias sectoriales que afectarán sin duda a la estrategia empresarial y al devenir de cada una de las entidades. Actualmente y hasta que se defina con mayor claridad el posicionamiento de cada Fundación en materia social, podemos decir que los ingresos por actividades propias provienen fundamentalmente de tres vías: (i) El patrimonio heredado al segregar la actividad financiera de la social. (ii) Los acuerdos alcanzados con el Banco en materia social. (iii) Los Montes de Piedad.

Para el primer caso, obtenemos que no todas las Fundaciones se han beneficiado del mismo volumen de activos en el reparto de sus bienes, existiendo $C A$ que antes de su transformación ya contaban con un mayor patrimonio histórico-artístico y de bienes inmuebles que otras. Por tanto, se obtendrán diferentes rentas en actividades como la explotación del patrimonio histórico-artístico, el alquiler de los bienes inmuebles o el aprovechamiento de los centros sociales, culturales, deportivos, etc.

En segundo lugar, existen entidades que han firmado acuerdos de colaboración en materia social con la entidad financiera y que les permite obtener flujos monetarios adicionales al reparto de dividendos, caso éste de las antiguas caixas catalanas respecto a BBVA. Finalmente, parece ser que los Montes de Piedad se consolidan como una alternativa para todas aquellas FO que no perciben dividendos de la entidad financiera. En este caso, si tomamos como referencia la memoria de Montes de Piedad para el ejercicio 2015, podemos observar que una vez materializado el proceso de reestructuración sectorial existen en España doce Montes de Piedad, siete vinculados a los Bancos y cinco a las Fundaciones.

De los cinco Montes de Piedad vinculados a las Fundaciones, cuatro pertenecen a FO sin participación accionarial dentro del Banco, existiendo casos como el de Montemadrid donde la principal vía de ingresos proviene de la actividad de los Montes. Por ello, la Fundación ha puesto en marcha 


\section{DE CAJAS DE AHORRO A SOCIEDADES CAPITALISTAS Y FUNDACIONES: UN NUEVO ENFOQUE EN LA GESTIÓN Y APLICACIÓN DE LA OBRA SOCIAL}

un plan de expansión para los próximos años que contempla la apertura de nuevas oficinas en diferentes provincias de España. Concretamente, en 2015 a la oficina de Madrid se unieron otras dos situadas en las localidades de Móstoles y Alicante ${ }^{12}$.

Por otra parte, si consideramos el reparto de dividendos como una de las principales fuentes de financiación de las nuevas Fundaciones, podemos observar que este flujo monetario no sólo está condicionado por la decisión del Banco de repartir beneficios, sino que la mayor parte de las Fundaciones resultantes del proceso de reestructuración cuentan con una escasa o nula participación en la entidad de crédito, caso éste de las CA que han sido intervenidas por el Banco de España y de aquellas cuya titularidad corresponde al Fondo de Reestructuración y Ordenación Bancaria (FROB).

\section{Tabla 3. Clasificación de las Fundaciones según su participación en la entidad financiera}

\begin{tabular}{|l|l|}
\hline Sin participación accionarial & $\begin{array}{l}\text { FO Afundación; FO Bancaja; FO Caja Ávila; FO Caja Mediterraneo; FO } \\
\text { Caja Rioja; FO Caja Segovia; FO Cajasur; FO España-Duero; FO La Caja } \\
\text { de Canarias; FO Montemadrid; FO lluro; FO Antigues Caixes Catalanes; } \\
\text { FO Catalunya-La Pedrera. }\end{array}$ \\
\hline & $\begin{array}{l}\text { FB CajaCírculo; FB Caja de Burgos; FB Caja de Ahorros de Santander y } \\
\text { Cantabria; FB Caja de Extremadura; FB Caja Navarra; FB Caja Canarias; } \\
\text { FO Caja Granada; FO Caixa Balears; FO Caja de Badajoz; FO Caja de } \\
\text { Ahorros Inmaculada de Aragón; FO Caja de Ahorros de Murcia; FO } \\
\text { Cajasol; FE Pinnae. }\end{array}$ \\
\hline Participación entre el 10-25\% & FB Caja Vital Kutxa; FB Caja Castilla la Mancha13. \\
\hline Participación entre el 25-50\% & FB Cajastur; FB Kutxa. \\
\hline Participación superior al 50\% & FB BBK; FB Ibercaja; FB la Caixa; FB Unicaja. \\
\hline Cajas de Ahorros & Caixa Ontinyent; Fundación Guillem Cifre de Colonya. \\
\hline
\end{tabular}

FUENTE: Elaboración propia.

En la Tabla 3 podemos apreciar la distribución accionarial tan heterogénea que se ha presentado tras la conversión de las CA en Fundaciones, existiendo trece entidades que no perciben beneficio alguno de la entidad financiera y otras doce en las que el reparto de dividendos es poco significativo, ya que poseen una participación inferior al 10\% entre el accionariado del Banco. Por tanto, si excluimos la actividad social de las dos CA, obtenemos que veinticinco de las treinta y tres Fundaciones se encuentran ante una tesitura en la que el flujo de dividendos es inexistente o muy exiguo como para constituir una base sólida que sirva para financiar el grueso de la política social.

12.- Véase Memoria 2015. Montes de Piedad. CECA. Madrid

13.- Está previsto que en 2017 Liberbank adquiera el 25\% que sigue en manos de la Fundación. 


\section{5.- Conclusiones}

La crisis económica puso de manifiesto una serie de desequilibrios que afectaban a los sistemas financieros de las principales economías mundiales. En España, este proceso de convulsión económica tuvo un mayor impacto en las CA, entidades en las que se evidenció una dudosa gestión de su modelo de negocio, un exceso de capacidad instalada, una elevada exposición al sector inmobiliario y en definitiva un fuerte endeudamiento. La elevada dependencia de financiación exterior como consecuencia de su naturaleza jurídica y la imposibilidad de acceder al mercado de capitales, hizo que algunas entidades tuvieran que ser rescatadas mediante ayudas públicas a través del FROB.

Para evitar situaciones similares que pudieran derivar en riesgos sistémicos, el Gobierno nacional a instancia de las recomendaciones dictadas por parte de algunos organismos europeos decidió elaborar una Ley transformadora del régimen jurídico y la actividad de las $C A$, desvinculándose de este modo de las actuaciones reformistas que había venido realizando hasta ese momento. Así, con la publicación de la LCAFB en diciembre de 2013 se da inicio a una nueva etapa en la que se separa la actividad financiera de la social y donde se da entrada a nuevos grupos de interés dentro de los órganos de decisión.

Una vez realizado el análisis normativo y sectorial, se ponen de manifiesto algunas cuestiones que afectan sustancialmente al modelo de CA que hemos conocido hasta que en 2010 se diera inicio al proceso de reestructuración. De este modo, se evidencia una profunda fragmentación del sector que influye en los elementos más característicos de este tipo de instituciones financieras: la OS y su Gobierno Corporativo.

Bajo el modelo tradicional, las CA presentaban un patrón similar en todo lo relacionado con su estructura de gobierno, distribución de excedentes, gestión de la OS y política social. Salvo casos muy concretos, en los órganos de decisión participaban los mismos grupos de interés y se destinaban cantidades prácticamente equivalentes a financiar la OS, siendo la Caja la encargada de financiar casi la totalidad de ésta que se aplicaba mediante la modalidad de OP u OC preferentemente en dos líneas de inversión social: Cultura y Tiempo Libre y Asistencia Social.

Con la entrada en vigor de la LCAFB surgen dos tipos de instituciones desde las que aplicar la política social (FB y FO), hallando diferencias significativas que afectan al modelo de negocio de cada una de ellas. Si consideramos en primer lugar la disposición de los órganos de decisión, se observan diferentes marcos normativos (Ley de Fundaciones para las FO y LCAFB para las FB) que pueden dar lugar a la formación de estructuras de gobierno heterogéneas entre las Fundaciones. En este caso, es en las FB donde se ha establecido una mayor vigilancia sobre la configuración de los órga- 


\section{DE CAJAS DE AHORRO A SOCIEDADES CAPITALISTAS Y FUNDACIONES: UN NUEVO ENFOQUE EN LA GESTIÓN Y APLICACIÓN DE LA OBRA SOCIAL}

nos de dirección, dejando un mayor vacío en las FO donde no se regulan ni los grupo de interés que pueden formar parte de los mismos, ni la transparencia de las entidades, quedando a la voluntad de éstas la publicación de informes anuales en los que se haga mención al gobierno corporativo y a la política de inversiones.

De igual modo, en el plano social encontramos grandes desigualdades dependiendo de la Fundación que tomemos en consideración, siendo a priori las entidades de mayor tamaño y con una participación significativa en la entidad de crédito las que cuentan con una mayor ventaja. Sin embargo, de las 34 Fundaciones vinculadas a los Bancos tan sólo 6 cuentan con una participación accionarial superior al $25 \%$ del capital, obteniendo el resto una aportación poco significativa o nula en la entidad financiera.

Este dato revela que existen unas pocas Fundaciones que perciben importantes flujos monetarios de las entidades de crédito y, en cambio, encontramos un elevado número de Fundaciones en las que el reparto de dividendos es nulo o poco significativo. Por tanto, cada entidad planteará estrategias diferenciadas en función de las necesidades de financiación de cada una de ellas, siendo previsible que sean las FB de mayor tamaño las que aborden los proyectos sociales más importantes.

Ante este contexto, las FO de menor tamaño deberán explotar otras ventajas competitivas como la especialización en zonas geográficas concretas, los acuerdos con instituciones público/privadas y las actividades propias desarrolladas a través de los centros sociales, culturales, deportivos, Patrimonio Histórico y Montes de Piedad. Por lo observado hasta ahora, algunas Fundaciones ya se han posicionado en este sentido, dando como resultado una OS financieramente mucho más pequeña que en periodo anterior al proceso de reestructuración sectorial, gestionada en su totalidad mediante la modalidad de OP y orientada hacia unos colectivos mucho más específicos. El fuerte gasto asistencial puede ser el resultado de optimizar las demandas sociales con una oferta de servicios que necesita ser eficiente ante un escenario en el que se han reducido considerablemente los flujos monetarios para este tipo de actividades.

Como futuras líneas de investigación sería interesante realizar un estudio de OS diferenciando entre FB y FO. La situación inicial ha dejado a algunas Fundaciones mejor posicionadas financieramente que a otras, lo que hace previsible que se planteen iniciativas específicas para cada caso en función de la fuente de ingresos que se desee potenciar. Este análisis puede hacerse extensivo a los órganos de gobierno, realizando una revisión individualizada sobre la estructura y composición de sus miembros. 


\section{Bibliografía}

AZOFRA, V. \& SANTAMARÍA, M. (2004): "El gobierno de las cajas de ahorros españolas", Universia Business Review, 2, 48-59.

BALADO, C. (2006): "La Obra Social de las Cajas, una paso siempre por delante en RSC", Papeles de Economía Española, 108, 144-160.

BOIX, A. \& UREÑA, J.A. (2006): "Obra benéfico-social de las cajas de ahorros y financiación de infraestructuras", Revista de Administración Pública, 170, 295-323.

CABEZA, L., MARTíNEZ, A. \& MARBELLA, F. (2010): "Análisis de la relación entre los resultados sociales y económicos de las cajas de ahorros españolas: Un caso de Responsabilidad Social Corporativa", Innovar-Social and Management Sciences Journal, 20, 33-46.

CLIMENT, S. (2012): "La caída de las cajas de ahorros españolas. Cuestión de rentabilidad, tamaño y estructura de propiedad", Estudios de Economía Aplicada, 30(2), 1-26.

CLIMENT, S. \& PAVÍA, J.M. (2014): "Determinantes y diferencias en la rentabilidad de Cajas y Bancos", Revista de Economía Aplicada, 65(22), 117-154.

EMPARANZA, A. (2015): Comentarios a la ley de cajas de ahorros y fundaciones bancarias, Thomson Reuters-Aranzadi.

ESCOBAR, B. \& GUZMÁN, I. (2010): "Eficiencia y cambio productivo en las cajas de ahorros españolas", CIRIEC-España, Revista de Economía Pública, Social y Cooperativa, 68, 183-202.

FONSECA, A.R. (2005): "El gobierno de las cajas de ahorros: influencia sobre la eficiencia y el riesgo", Universia Business Review, 8, 24-37.

FONSECA, A.R. \& GONZÁLEZ, F. (2005): "Cambios en el gobierno de las Cajas de Ahorros y nivel de riesgo. Efecto de las legislaciones autonómicas", Revista Española de Financiación y Contabilidad, 125(34), 395-421.

GARCÍA-CESTONA, M. \& SURROCA, J. (2008): "Multiple goals and ownership structure: Effects on the performance of Spanish savings banks", European Journal of Operational Research, 187(2), 582-599.

GUZMÁN, I. \& REVERTE, C. (2008): "Productivity and efficiency change and shareholder value: evidence from the Spanish banking sector", Applied Economics, 40(15), 2033-2040.

ILLUECA, M., NORDEN, L. \& UDELL, G. (2011): "Liberalization, bank governance, and risk taking", American Economic Association 2012 Meetings Paper. 
MARTíNEZ, A., CABEZA, L. \& MARBELLA, F. (2013): "Responsabilidad social corporativa y resultado financiero: evidencia sobre la doble dirección de la casualidad en el sector de las Cajas de Ahorros", Cuadernos de Economía y Dirección de la Empresa, 16(1), 54-68.

MARTÍNEZ, A. (2014): "La Ley 26/2013, de Cajas de Ahorro y Fundaciones bancarias: ¿hemos aprendido del pasado?", Revista de Derecho de Sociedades, 42, 309-320.

MAUDOS, J. (2001): "Rentabilidad, estructura de mercado y eficiencia en la banca", Revista de Economía Aplicada, 25, 193-207.

MELLE, M. \& MAROTO, J.M. (1999): "Una aplicación del gobierno de empresas: incidencias de las Administraciones Públicas en las decisiones asignativas de las cajas de ahorros españolas", Revista Europea de Economía de Empresa, 8, 9-39.

PÉREZ, A. \& RODRÍGUEZ, I. (2012): "La imagen de Responsabilidad Social Corporativa en un contexto de crisis económica: El caso del sector financiero en España", Universia Business Review, 1(33), 14-29.

TORRES, J., RETOLAZA, J. \& SAN-JOSÉ, L. (2012): “Gobernanza multifiduciaria de stakeholders: Análisis comparado de la eficiencia de bancos y cajas de ahorros", REVESCO, Revista de Estudios Cooperativos, 108, 152-172.

TRUJILLO-PONCE, A. (2013): "What determines the profitability of banks? Evidence from Spain", Accounting and Finance, 53(2), 561-586.

UREÑA, J.A. (2015): "Crisis y transformación del sistema bancario español", Revista General de Derecho Administrativo, núm. 40. 
